\title{
Review Article \\ The Role of Endoplasmic Reticulum Stress in Cardiovascular Disease and Exercise
}

\author{
Junyoung Hong, ${ }^{1}$ Kwangchan Kim, ${ }^{1}$ Jong-Hee Kim, ${ }^{2}$ and Yoonjung Park ${ }^{1}$ \\ ${ }^{1}$ Laboratory of Integrated Physiology, Department of Health and Human Performance, University of Houston, \\ Texas, TX 77204, USA \\ ${ }^{2}$ Department of Physical Education, Hanyang University, Seoul, Republic of Korea \\ Correspondence should be addressed to Yoonjung Park; ypark10@uh.edu
}

Received 5 May 2017; Accepted 6 July 2017; Published 10 August 2017

Academic Editor: Francesco Fugetto

Copyright (C) 2017 Junyoung Hong et al. This is an open access article distributed under the Creative Commons Attribution License, which permits unrestricted use, distribution, and reproduction in any medium, provided the original work is properly cited.

Endoplasmic reticulum (ER) stress, which is highly associated with cardiovascular disease, is triggered by a disturbance in ER function because of protein misfolding or an increase in protein secretion. Prolonged disruption of ER causes ER stress and activation of the unfolded protein response (UPR) and leads to various diseases. Eukaryotic cells respond to ER stress via three major sensors that are bound to the ER membrane: activating transcription factor 6 (ATF6), inositol-requiring protein $1 \alpha$ (IRE1 $\alpha$ ), and protein kinase RNA-like ER kinase (PERK). Chronic activation of ER stress causes damage in endothelial cells (EC) via apoptosis, inflammation, and oxidative stress signaling pathways. The alleviation of ER stress has recently been accepted as a potential therapeutic target to treat cardiovascular diseases such as heart failure, hypertension, and atherosclerosis. Exercise training is an effective nonpharmacological approach for preventing and alleviating cardiovascular disease. We here review the recent viewing of ER stress-mediated apoptosis and inflammation signaling pathways in cardiovascular disease and the role of exercise in ER stress-associated diseases.

\section{Introduction}

The endoplasmic reticulum (ER) plays an essential role in controlling various intracellular physiological functions, including protein translocation, protein folding, calcium homeostasis, and lipid biosynthesis [1], by stimulating the signaling networks that control either cell survival or death when ER transmembrane sensors detect unfolded and misfolded proteins. Various pathophysiological conditions disturb ER homeostasis and can lead to the chronic activation of the unfolded protein response (UPR) in ER lumen, which causes ER stress. Prolonged ER stress activates three ER transmembrane sensors and initiates UPR signaling, which induces oxidative stress, inflammation, and apoptotic response. It aggravates neurodegenerative disease, cancer, metabolic disease, and kidney disease [2,3] and is highly associated with cardiovascular diseases such as cardiac hypertrophy, heart failure, atherosclerosis, and ischemic heart disease [4-7]. In particular, ER stress-induced endothelial cell (EC) damage and dysfunction exert the negative influences on cardiovascular diseases through apoptosis and inflammation [8].

It has been well established that exercise training can improve EC function and decrease the risk of cardiovascular diseases by increasing nitric oxide (NO) bioavailability, diminishing the volume of plaque and vascular viscosity, and increasing both diastolic coronary perfusion and vascular reactivity $[9,10]$. Numerous studies report that exercise could effectively improve various ER stress-related pathologies such as obesity, diabetes, neurodegenerative disease, hypoxia, and sarcopenia in skeletal muscle, liver, brain, and cardiovascular systems [11-17]. In this review, we focus on the mechanism of ER stress-mediated apoptosis and inflammation in cardiovascular disease, and the effect of exercise on ER stressassociated diseases.

\section{ER Stress and UPR Signaling}

The ER is an intracellular organelle covered by an extensive membrane network and is present in all eukaryotic cells. It 


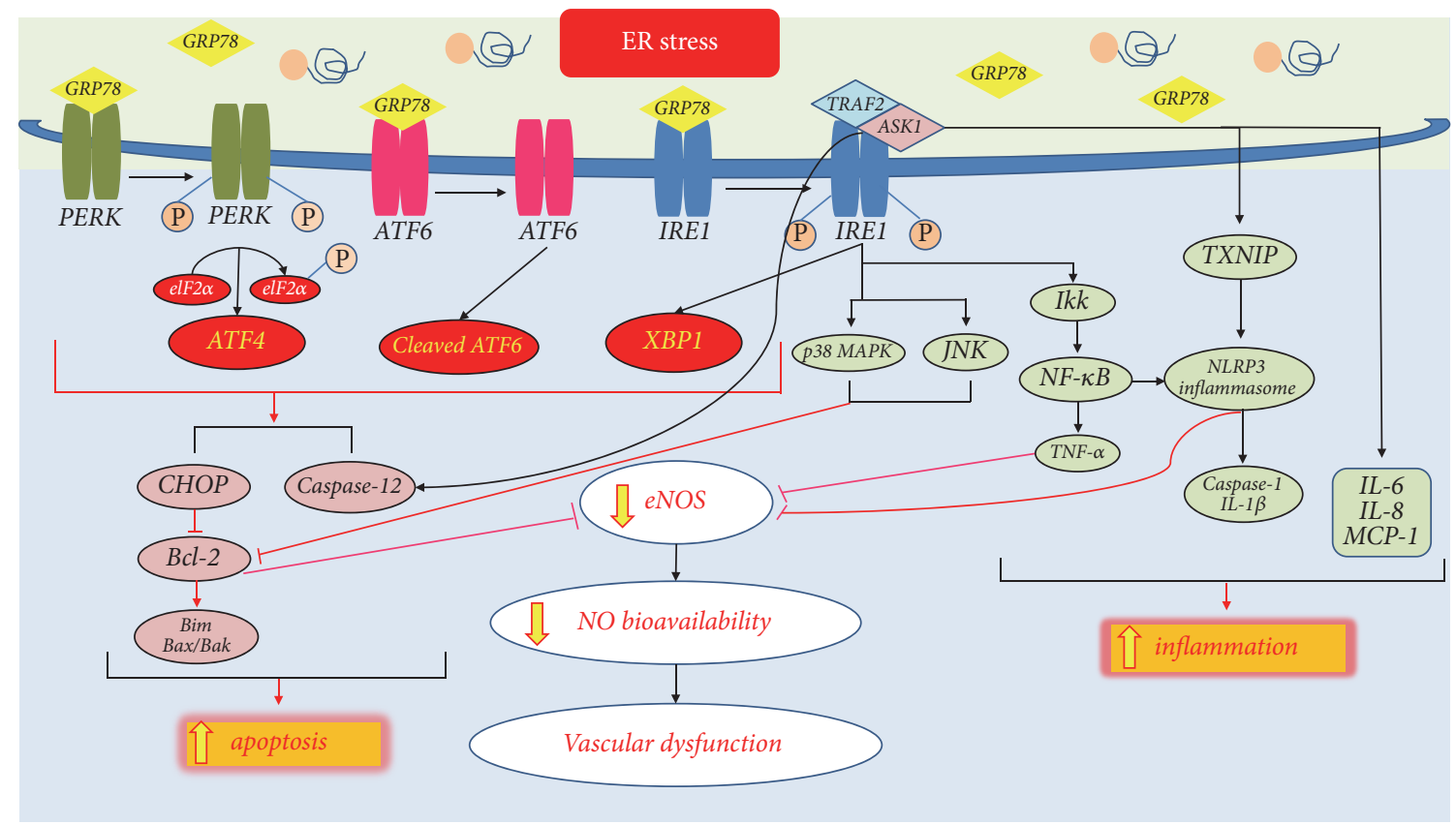

FIGURE 1: ER stress-mediated apoptosis and inflammation in cardiovascular disease. When prolonged ER stress occurs, activation of CHOPmediated and JNK/p38 MAPK-mediated apoptosis signaling pathways regulate the Bcl-2 family, which controls the balance of pro/antiapoptotic signaling, and ER stress-induced apoptosis signaling dampens eNOS expression with the increased oxidative stress. The IRE1 $\alpha /$ TRAF2/ASK1 complex also activates caspase-12 and ultimately induces cell apoptosis. Furthermore, ER stress-mediated NF- $\kappa$ B-IKK activates NLRP3 inflammasome and mediates eNOS activity. Excessive ER stress and activation of the IRE1 $\alpha /$ TRAF2/ASK1 pathway increase TXNIP activation that subsequently induces NLRP3 inflammasome and reduced eNOS activity. The IRE1 $\alpha /$ TRAF2/ASK1 complex also contributes to the expression of proinflammations that stimulate NLRP3 inflammasome-induced caspase- 1 and IL- $1 \beta$ and UPR-mediated IL-6. ER stress also activates IL-8, MCP-1, and TNF- $\alpha$ expression. Ultimately, the accumulation of ER stress-mediated apoptotic and inflammatory responses decreases the eNOS activity which diminishes NO bioavailability and causes vascular dysfunction. The yellow arrows represent ER stress-mediated pathway.

is the major site of protein synthesis, protein folding, protein transport, lipid production, and calcium storage [18]. Multiple biological insults such as calcium and redox imbalance, viral infection, oxidative stress, and hyperlipidemia lead to perturbation of ER homeostasis, which consequently initiates the accumulation of unfolded proteins in the ER lumen that is called ER stress $[19,20]$.

The UPR, which is chiefly responsible for determining cellular death or survival responses in accordance with ER stress, is designed to restore ER homeostasis by activating ER-associated protein degradation (ERAD), autophagy, and cell survival signals [21]. Excessive ER stress, however, activates UPR and promotes ER stress-associated signaling cascades that stimulate apoptosis and inflammatory signaling pathway [22, 23] (Figure 1). The UPR generally senses the misfolded proteins in the ER and activates each signaling pathway through three sensors bound to the ER membrane (Figure 2): PKR-like eukaryotic initiation factor $2 \alpha$ kinase (PERK), inositol-requiring protein 1 (IRE1), and activating transcription factor-6 (ATF6) $[24,25]$. In unstressed conditions, the transmembrane proteins bind to glucose-regulated protein 78 (GRP78 or $\mathrm{BiP}$ ), a molecular chaperone that inhibits the activities of these transmembrane proteins [25]. When ER stress occurs, GRP78 dissociates from the three sensors and initiates the UPR. By the onset of ER stress, the PERK signaling pathway decreases protein synthesis by reducing protein load in ER lumen and the activated PERK phosphorylates eukaryotic initiation factor- $\alpha$ (elF2 $\alpha)$. Eventually, the process induces cell death via certain gene families including activating transcription factor-4 (ATF4) and C/EBP homologous protein (CHOP) [1]. IRE1 promotes the activation of major inflammatory response factors in signaling pathways and induces splicing of a transcriptional regulatory factor in the bZip family, called X-box binding protein-1 (XBP-1), through its site-specific endoribonuclease (RNase) function [26]. When ATF6 is released by BiP, it is translocated and activated after proteolysis in the Golgi apparatus [27]. In turn, ATF6 activates the synthesis of ER chaperones and XBP-1. Therefore, the three branches of UPR signaling (ATF4, XBP-1, and ATF6) are highly activated to regulate the transcription of ER chaperones in ER stress that blocks protein synthesis and decreases protein folding capacity $[28,29]$.

\section{ER Stress-Mediated Apoptosis in Cardiovascular Disease}

ER stress has been highlighted as an important regulator of cardiovascular diseases [30-32]. The endothelium is the crucial site to maintain vascular homeostasis and control 


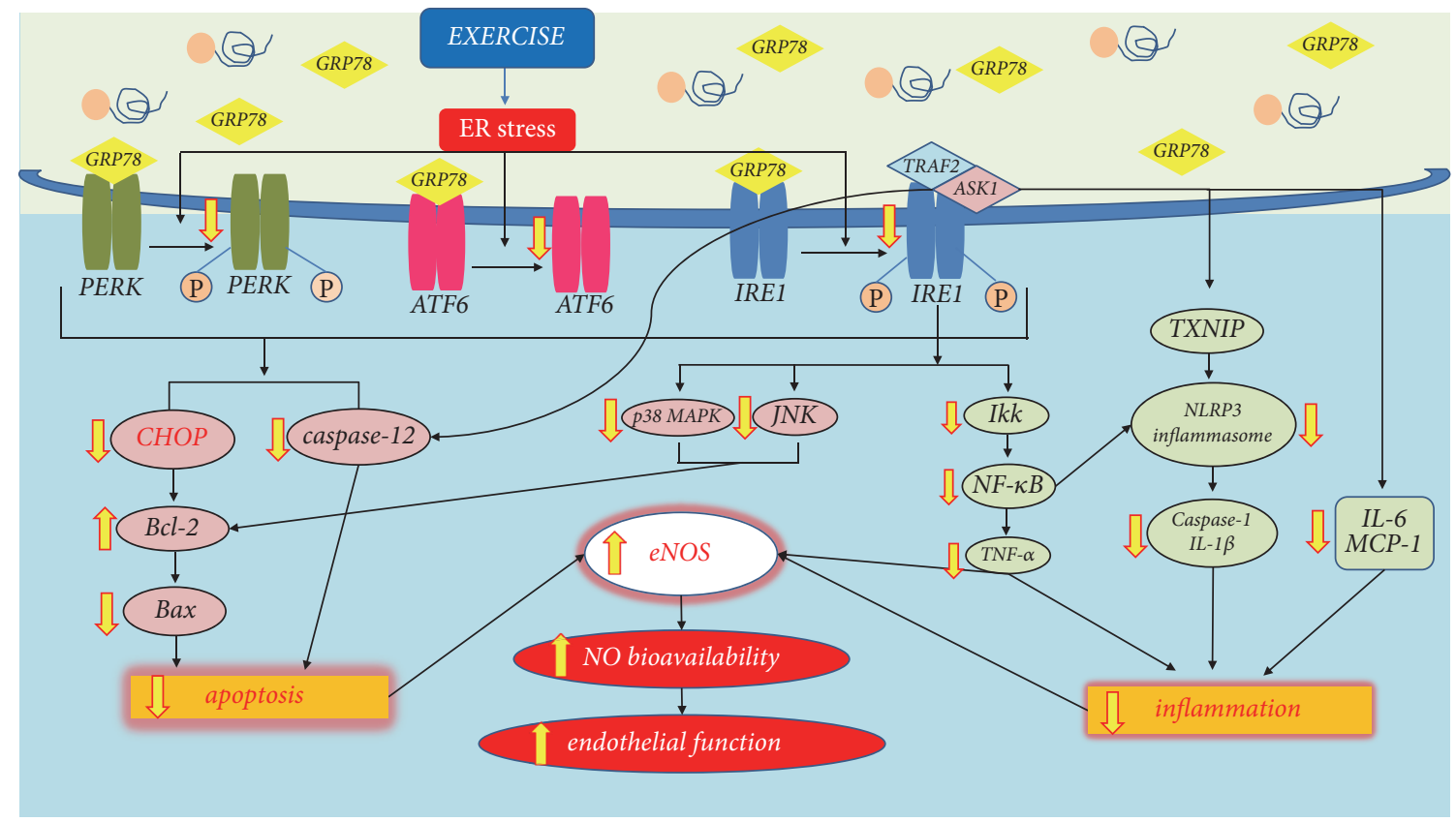

FIGURE 2: The Chronic effect of exercise on the ER stress-associated apoptosis and inflammation. Regular exercise reduces phosphorylation of the three ER stress sensors and inhibits or decreases ER stress-associated apoptosis and inflammation signaling pathways. Exercise training ameliorates ER stress-mediated CHOP signaling, in turn reducing apoptosis. Exercise training also reduces IRE1 $\alpha$-mediated p38 MAPK/JNK and NF- $\kappa$ B-associated inflammasome regulating the expression of caspase-1, IL-1 $\beta$, IL-6, and MCP-1, mitigates the IRE1 $\alpha /$ TRAF2/ASK1 complex that links to caspase-12, and mediates apoptosis in cardiovascular disease. As a result, these signaling pathways systemically mediate eNOS expression through the decreased apoptosis and inflammation, which increase NO bioavailability and improve endothelial function. The yellow arrows represent the exercise-induced changes in ER stress-mediated apoptosis and inflammatory pathway.

vascular reactivity via endothelium-derived relaxing factors (EDRFs) [33]. Endothelial dysfunction is the initial response in many cardiovascular and metabolic diseases. CHOP, the most widely investigated biomarker involved in ER stressassociated apoptotic signaling in cardiovascular disease, is regulated by anti- and proapoptotic protein of Bcl-2 family [32]. In atherosclerosis, the UPR fails to control misfolded proteins in the ER and increases the expression of CHOP with a progression of atherosclerosis in the aorta. Eventually, it activates $\mathrm{CHOP}$-induced apoptosis signaling and further responses [6, 30-32, 34]. PERK/ATF4 and ATF6-dependent pathways regulate the CHOP-mediated proapoptotic bZIP transcriptional factor and IRE1-dependent apoptotic signaling is activated through various processes $[34,35]$. IRE1 interacts with the TNF receptor-associated factor (TRAF) 2; in turn, the complex of IRE1 and TRAF2 are associated with apoptosis signal-regulating kinase 1 (ASK1), which activates both the c-Jun N-terminal kinase (JNK) and p38 mitogenactivated protein kinases (MAPK) $[35,36]$.

The Bcl-2 family of genes is an important apoptotic factor for controlling the balance of proapoptotic and antiapoptotic signals when both CHOP and IRE1 are activated [37] (Figure 2). The Bcl-2 family includes both antiapoptotic and proapoptotic members that mediate the crosstalk between the ER and mitochondria. Bax and Bak are the most well-known proapoptotic members and $\mathrm{Bcl}-2$ and $\mathrm{Bcl}-\mathrm{x}$ are the most widely known antiapoptotic members of the Bcl-2 family. Activating the proapoptotic proteins on the mitochondrial membrane releases cytochrome-c and causes subsequent apoptosis induction [38]. Because of the decline of mitochondrial function in ECs via the CHOP-mediated proapoptotic proteins [39] and the interrupted calcium homeostasis [40], the levels of reactive oxygen species (ROS) and nicotinamide adenine dinucleotide phosphate (NADPH) are increased in ECs in atherosclerosis $[41,42]$. It is unclear whether NADPH oxidases act as upstream or downstream of ER stress-induced cardiovascular dysfunction. Recent studies report that ER stress-associated oxidative stress and inflammation suppress the production of $\mathrm{NO}$ and endothelial nitric oxide synthase (eNOS) activity, both of which protect ECs [8,30, 43-45]. The ER stress-induced activation of JNK causes oxidative stress and vascular endothelial dysfunction through repression of eNOS activity [46, 47].

The caspase-12 protein regulates ER stress-induced apoptosis signaling [31, 48] (Figure 2). Two pathways activate procaspase-12. First, the elevated $\mathrm{Ca}^{2+}$ level in the cytoplasm activates procaspase-12 in the ER membrane to form caspase12; the process requires calpain that induces activation of caspase- 9 and finally activates caspase- 3 and apoptosis [49]. Second, during ER stress, TRAF2 dissociates from the complex of TRAF2/procaspase-12 located in the ER membrane; the process activates caspase- 12 and recruits the IRE1/JNK/TRAF2 complex that modulates ASK1 that in turn phosphorylates JNK and induces cell apoptosis [50]. Ischemia/reperfusion ( $\mathrm{I} / \mathrm{R})$ showed the elevated level of caspase-12 and ER stress markers in myocardium [46, 51-53], 
and the expression of caspase- 12 and cleaved caspase- 3 were abnormally altered in the pathological cardiac hypertrophy in rodent model $[54,55]$.

\section{ER Stress-Mediated Inflammation in Cardiovascular Diseases}

Inflammation is an immunological response to infection or tissue damage and protects the body from such injuries. Chronic inflammation aggravates tissue damage, and it can influence the development of cardiac hypertrophy, heart failure, coronary artery diseases, and atherosclerosis $[56,57]$. ER stress and inflammation signaling pathways are connected through various mechanisms that can induce cardiovascular disease (Figure 2). In atherosclerosis, increased PERK and IRE1/TRAF2 and accumulated ROS activate and augment inflammatory response [58]. PERK and IRE1/TRAF2 complex can recruit I $\kappa B$ kinase (IKK), which phosphorylates $\mathrm{I} \kappa \mathrm{B}$, resulting in the degradation of $\mathrm{I} \kappa \mathrm{B}$ and the nuclear translocation of NF- $\kappa \mathrm{B}$ [59]. The NF- $\kappa \mathrm{B}-\mathrm{IKK}$ pathway is a key regulator in the induction of inflammatory mediators [59, 60]. Recent studies report that ATF6 also interacts with NF$\kappa \mathrm{B}-\mathrm{IKK}$, indicating that all three sensors of ER stress (PERK, IRE1, and ATF6) can induce specific inflammatory responses through the UPR [61]. Induction of the UPR at the cellular level increases the expression of inflammatory molecules, including IL-8, IL-6, MCP-1, and TNF- $\alpha$, thereby inducing atherosclerosis [62]. Other studies report that TNF- $\alpha$ and NF$\kappa \mathrm{B}$ signaling through the IKK pathway can provoke coronary arteriolar dysfunction [63]. TNF- $\alpha$ reduces the bioavailability of NO by increasing the activity of NADPH and downregulating eNOS [63-66]. IL-1 $\beta$ and TNF- $\alpha$ are associated with the increased expression of vascular cell adhesion molecule 1 (VCAM-1) and intercellular cell adhesion molecule 1 (ICAM1) [67]. IL-1 $\beta$ and TNF- $\alpha$ also stimulate apoptosis through promoting the expression of caspase family and inducible NOS (iNOS) in ECs [68-70].

Other recent studies report that NF- $\kappa \mathrm{B}$, an immunological mediator, plays a vital role in controlling the NODlike receptor family, pyrin domain containing 3 (NLRP3) inflammasome [71-73]. Notably, IRE1 induces an elevation of thioredoxin-interacting protein (TXNIP); the elevated TXNIP promotes inflammation and cell apoptosis by activating NLRP3 inflammasome that in turn activates caspase-1 to induce the secretion of IL-1 $\beta$ [73-75] (Figure 2). Activated caspase- 1 is usually observed in ruptured plaques; the survival rate of patients who have high plasma levels of caspase-1 is much lower than the individuals with a normal level of plasma caspase-1 [76]. In addition, NLRP3 inflammasomeinduced increases in IL-1 $\beta$ are known to upregulate proinflammatory and proapoptotic genes in ECs [77]. ER stressassociated TXNIP/NLRP3 signaling is activated in a high concentration of palmitate-treated endothelial cells and in the high fat diet-fed mice aorta. It induces the endothelial dysfunction through enhanced oxidative stress and reduced eNOS expression [78]. Pharmacological treatment using AMP-activated protein kinase (AMPK) can improve the endothelial dysfunction caused by the activation of ER stressassociated TXNIP/NLRP3; AMPK improves mitochondrial morphology and endothelial dysfunction by repressing mitochondrial ROS-associated ER stress-dependent activation of the TXNIP/NLRP3 inflammasome [79].

Perturbation of ER stress and UPR leads to a toxic intracellular accumulation of ROS, a possible cause of ER stress-associated inflammation $[1,80]$. Alteration of PERK and ATF4 signaling is responsible for ER stress-associated redox imbalance and affects the disulfide bond formation (ERO1, endoplasmic reticulum oxidoreductin; PDI, protein disulfide isomerase) that influences antioxidant activation in ER [2]. A PERK-mediated Nrf2 cascade impairs the antioxidant process that stimulates $\mathrm{I} \kappa \mathrm{B} / \mathrm{NF}-\kappa \mathrm{B}$ signaling, resulting in increased inflammatory response of IL- 6 and TNF- $\alpha$ expression during ER stress $[1,60,81]$.

\section{ER Stress and Exercise}

Regular exercise is considered an effective tool to prevent and reduce the risk of cardiovascular disease [33]. Endothelial dysfunction with the reduction of NO bioavailability is a common symptom of hypertension, obesity, heart failure, and atherosclerosis [64, 82-84]. Exercise training provides the numerous positive effects on endothelial dysfunction and helps maintain cardiovascular homeostasis through an increase in antioxidative response and a reduction of inflammatory cytokines expression $[10,56,82,85,86]$. The beneficial effect of exercise on ER stress, however, will depend on the modality and duration of exercise. There is still insufficient data to establish the definitive effects of exercise training on ER stress-associated cardiovascular disease. Therefore, this section discusses the overall exercise effects on ER stressassociated diseases.

Chronic aerobic exercise training shows the diverse patterns in the expression of ER stress markers (Figure 3). Treadmill exercise can improve cardiac function and reduce cardiac infarction by attenuating the expression of GRP78, DERLIN1, p-PERK, p-eIF2 $\alpha$, ATF4/6, XBP1, CHOP, and cleaved caspase-3 $[11,87]$. Treadmill exercise has been shown to ameliorate ER stress (p-eIF2 $\alpha$, ATF3, and ATF4) and endothelial dysfunction (conduit and resistance vessels) in diabetic mice through a PPAR $\gamma$-dependent mechanism with an increase in NO bioavailability [88]. The expressions of ER stress markers (GRP78, p-PERK, p-elF2 $\alpha$, p-IRE1 $\alpha$, p-ATF6 $\alpha$, p-sXBP1, and $\mathrm{p}-\mathrm{CHOP}$ ), ER stress-induced apoptotic proteins (procaspase3, 12, p-JNK, Bax, and Bcl-2), and ER stress-induced inflammatory cytokines (NLRP3/IL-1 $\beta$ and proinflammatory cytokines) were decreased after treadmill exercise in an obese rodent $[12,13,15]$. Swimming exercise reduced p-PERK, peIF $2 \alpha$, JNK, IkB $\alpha$, and NF- $\kappa$ B in an obese mice model [13, $15,89]$. However, one study has reported that treadmill exercise training elevated UPR response (PERK, IREl $\alpha$ ) with a reduction of inflammation (IL-6 and MCP-1) in high fat dietinduced mice model [90]. After 3 months of combination of aerobic and resistance exercises training, mRNA and protein levels of GRP78, p-IRE1 $\alpha$, and p-eIF $2 \alpha$ were decreased in subcutaneous adipose tissue and peripheral blood mononuclear cells (PBMCs) in obese adult subjects [16].

After mice completed 8 weeks of uphill and downhill running, downhill running increased the BiP, ATF6, 


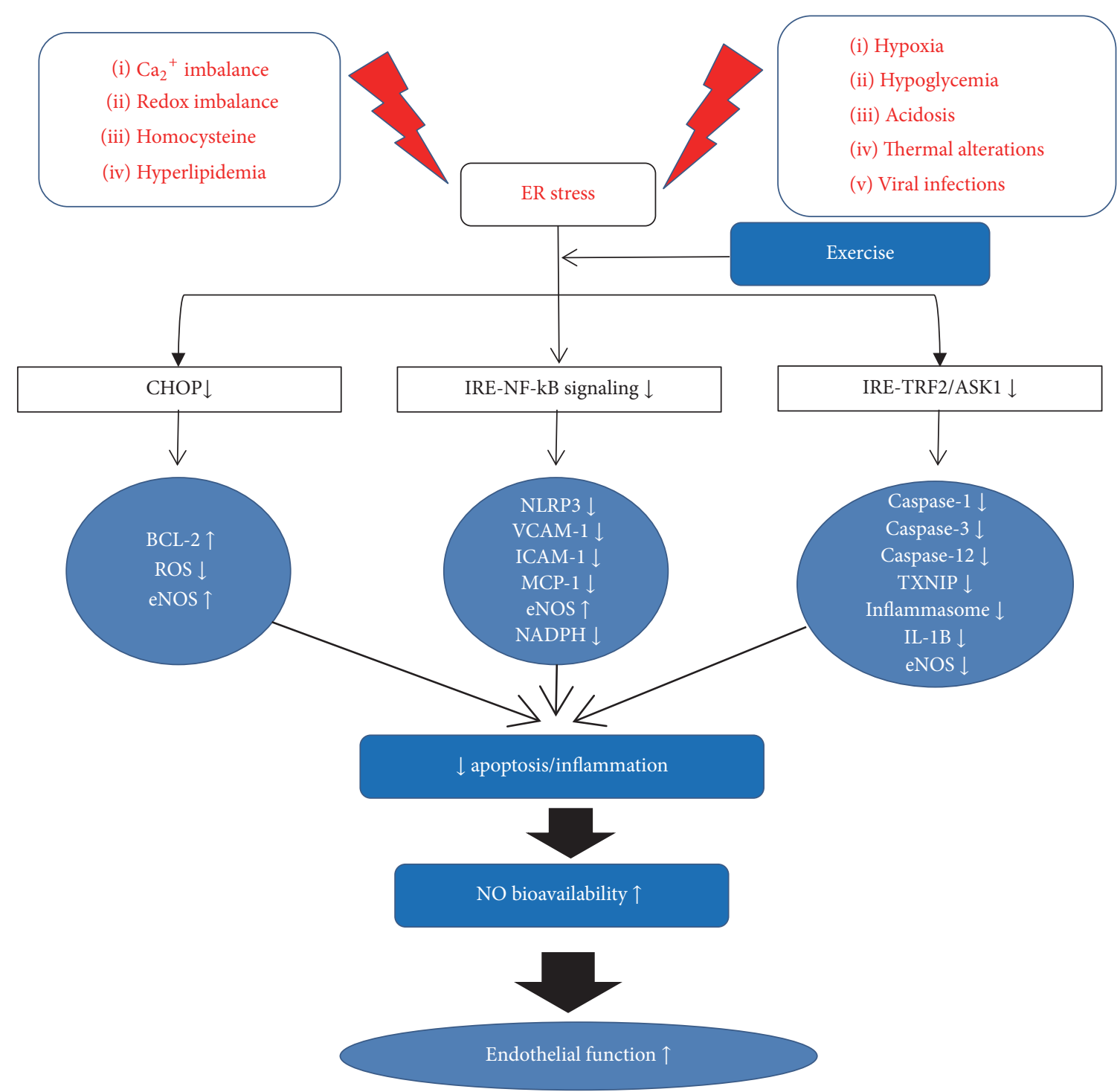

FIGURE 3: Schematic diagram of the chronic effect of exercise on ER stress and endothelial function. ER stress-mediated apoptosis and inflammatory responses are altered by exercise training; (1) CHOP-mediated, (2) IRE1 $\alpha / \mathrm{NF}-\kappa \mathrm{B}$ complex-associated, and (3) IRE1 $\alpha / \mathrm{TRAF} 2 / \mathrm{ASK} 1-$ associated gene/protein expression of apoptosis and inflammation are downregulated and eventually increase endothelial function by the elevated NO bioavailability.

p-IRE1- $\alpha$, and p-PERK expression in the extensor digitorum longus (EDL) muscle compared with uphill running. The finding suggests that eccentric contraction-induced muscle injuries stimulated the ER stress [91]. Furthermore, GRP78 was significantly elevated in both low and high intensity acute aerobic exercise training in rat model and adolescents with type 2 diabetic patients [92, 93]. ER stress-related gene and protein expression (p-PERK, XBP-1s, p-eIF- $\alpha$ ), respectively, increased but no changes of apoptosis signaling were found in high fat diet-induced obese and muscular dystrophy mice models after 3 or 7 weeks of voluntary exercise $[14,94]$

Short-term aerobic exercise is not sufficient to induce ER stress adaptation in animal models. GRP78, CHOP, cleaved/procaspase-12, and ATF3 were not altered after 5 days of aerobic training in rat model, and ER stress-related protein expression was not affected by one-day swimming exercise in TLR4-deficient mice [95, 96]. Acute exercise changes the expression of UPR/ER stress signaling. CHOPmediated apoptosis signaling ( $\mathrm{Bax}, \mathrm{Bcl}-2$, and caspase-3, 12) was activated with the elevated intracellular $\mathrm{Ca}^{2+}$ after rats completed one bout of swimming exercise [96]. In contrast, acute resistance exercise by untrained men elevated UPR signaling but did not change CHOP expression [97].

Altogether, exercise training has been shown to mitigate all three ER stress markers, ER stress-mediated inflammation, and apoptosis in metabolic and chronic disease model. However, published ER stress-associated studies on skeletal muscle showed that ER stress markers such as GRP78, pPERK, p-IRE1, and CHOP were activated and elevated after 
different types of aerobic and resistance training [91, 9799]. Interestingly, additional studies reported that the mice group which had previous training showed the decreased activation of UPR and CHOP genes compared to untrained mice after equal amount of treadmill exercise [98]. Also, the elevated mRNA levels of UPR genes (Bip, ATF4, and $\mathrm{XBP} 1$ s) and $\mathrm{CHOP}$, as well as CHOP protein expression after initial response to chronic contractile activity (CCA), were attenuated with repeated bouts of CCA in rats [100].

Therefore, these study results suggested that initial response to exercise training induces ER stress and activate UPR signaling. However, after prolonged exercise training, these increased markers are alleviated, which suggested that the activated UPR signaling is induced to acclimate to exercise training for cell survival and adaptation.

\section{Conclusion}

ER, an essential organelle for cell homeostasis, plays a central role in cell death and survival signaling. While myriad studies have demonstrated that chronic ER stress is one of the major contributors to cardiovascular disease, it is not precisely understood how ER stress modulates endothelium-dependent vascular function, especially NO signaling pathway. Exercise training elicits a beneficial effect on the endothelial function by increasing the antioxidative/inflammatory response in cardiovascular system. Additional well-designed, controlled studies of the exercise effect on ER stress-mediated cardiovascular disease and the mechanisms that trigger ER stress-associated cardiovascular dysfunction will close the existing knowledge gap and lead to the development of new therapeutic targets.

\section{Conflicts of Interest}

The authors declare that they have no conflicts of interest.

\section{References}

[1] G. S. Hotamisligil, "Endoplasmic reticulum stress and the inflammatory basis of metabolic disease," Cell, vol. 140, no. 6, pp. 900-917, 2010.

[2] B. Bhandary, A. Marahatta, H.-R. Kim, and H.-J. Chae, "An involvement of oxidative stress in endoplasmic reticulum stress and its associated diseases," International Journal of Molecular Sciences, vol. 14, no. 1, pp. 434-456, 2013.

[3] R. Sano and J. C. Reed, "ER stress-induced cell death mechanisms," Biochimica et Biophysica Acta-Molecular Cell Research, vol. 1833, no. 12, pp. 3460-3470, 2013.

[4] M. Kassan, M. Galán, M. Partyka et al., "Endoplasmic reticulum stress is involved in cardiac damage and vascular endothelial dysfunction in hypertensive mice.," Arteriosclerosis, thrombosis, and vascular biology, vol. 32, no. 7, pp. 1652-1661, 2012.

[5] J. Padilla and N. T. Jenkins, "Induction of endoplasmic reticulum stress impairs insulin-stimulated vasomotor relaxation in rat aortic rings: role of endothelin-1," Journal of Physiology and Pharmacology, vol. 64, no. 5, pp. 557-564, 2013.

[6] S. Shinozaki, T. Chiba, K. Kokame, T. Miyata, E. Kaneko, and K. Shimokado, "A deficiency of herp, an endoplasmic reticulum stress protein, suppresses atherosclerosis in ApoE knockout mice by attenuating inflammatory responses," PLoS ONE, vol. 8, no. 10, Article ID e75249, 2013.

[7] Expert Panel on Detection Evaluation and Treatment of High Blood Cholesterol in Adults, "Executive summary of the third report of the national cholesterol education program (NCEP) expert panel on detection, evaluation, and treatment of high blood cholesterol in adults (adult treatment panel III)," Journal of the American Medical Association, vol. 285, no. 19, pp. 24862497, 2001.

[8] I. Tabas, "The role of endoplasmic reticulum stress in the progression of atherosclerosis," Circulation Research, vol. 107, no. 7, pp. 839-850, 2010.

[9] D. Skrypnik, P. Bogdanski, E. Madry, D. Pupek-Musialik, and J. Walkowiak, "Effect of physical exercise on endothelial function, indicators of inflammation and oxidative stress," Pol Merkur Lekarski, vol. 36, pp. 117-121, 2014.

[10] Y. Park, F. W. Booth, S. Lee, M. J. Laye, and C. H. Zhang, "Physical activity opposes coronary vascular dysfunction induced during high fat feeding in mice," The Journal of Physiology, vol. 590, no. 17, pp. 4255-4268, 2012.

[11] G. Bourdier, P. Flore, H. Sanchez, J.-L. Pepin, E. Belaidi, and C. Arnaud, "High-intensity training reduces intermittent hypoxiainduced ER stress and myocardial infarct size," American Journal of Physiology - Heart and Circulatory Physiology, vol. 310, no. 2, pp. H279-H289, 2016.

[12] M. Cai, H. Wang, J.-J. Li et al., "The signaling mechanisms of hippocampal endoplasmic reticulum stress affecting neuronal plasticity-related protein levels in high fat diet-induced obese rats and the regulation of aerobic exercise," Brain, Behavior, and Immunity, vol. 57, pp. 347-359, 2016.

[13] G. da Luz, M. J. S. Frederico, S. da Silva et al., "Endurance exercise training ameliorates insulin resistance and reticulum stress in adipose and hepatic tissue in obese rats," European Journal of Applied Physiology, vol. 111, no. 9, pp. 2015-2023, 2011.

[14] J. J. Hulmi, J. Hentilä, K. C. DeRuisseau et al., "Effects of muscular dystrophy, exercise and blocking activin receptor IIB ligands on the unfolded protein response and oxidative stress," Free Radical Biology and Medicine, vol. 99, pp. 308-322, 2016.

[15] E.-B. Kang, I.-S. Kwon, J.-H. Koo et al., "Treadmill exercise represses neuronal cell death and inflammation during $\mathrm{A} \beta$ induced ER stress by regulating unfolded protein response in aged presenilin 2 mutant mice," Apoptosis, vol. 18, no. 11, pp. 1332-1347, 2013.

[16] A. Khadir, S. Kavalakatt, J. Abubaker et al., "Physical exercise alleviates ER stress in obese humans through reduction in the expression and release of GRP78 chaperone," Metabolism: Clinical and Experimental, vol. 65, no. 9, pp. 1409-1420, 2016.

[17] E. Passos, A. Ascensão, M. J. Martins, and J. Magalhães, "Endoplasmic reticulum stress response in non-alcoholic steatohepatitis: the possible role of physical exercise," Metabolism, vol. 64, no. 7, pp. 780-792, 2015.

[18] P. Walter and D. Ron, "The unfolded protein response: from stress pathway to homeostatic regulation," Science, vol. 334, no. 6059, pp. 1081-1086, 2011.

[19] I. Kim, W. Xu, and J. C. Reed, "Cell death and endoplasmic reticulum stress: disease relevance and therapeutic opportunities," Nature Reviews Drug Discovery, vol. 7, no. 12, pp. 1013-1030, 2008.

[20] D. J. Maly and F. R. Papa, "Druggable sensors of the unfolded protein response," Nature Chemical Biology, vol. 10, no. 11, pp. 892-901, 2014. 
[21] S. Lenna, R. Han, and M. Trojanowska, "Endoplasmic reticulum stress and endothelial dysfunction," IUBMB Life, vol. 66, no. 8, pp. 530-537, 2014.

[22] B. Luo and A. S. Lee, "The critical roles of endoplasmic reticulum chaperones and unfolded protein response in tumorigenesis and anticancer therapies," Oncogene, vol. 32, no. 7, pp. 805-818, 2013.

[23] G. Zhu and A. S. Lee, "Role of the unfolded protein response, GRP78 and GRP94 in organ homeostasis," Journal of Cellular Physiology, vol. 230, no. 7, pp. 1413-1420, 2014.

[24] M. Schröder and R. J. Kaufman, "The mammalian unfolded protein response," Annual Review of Biochemistry, vol. 74, pp. 739-789, 2005.

[25] A. Bertolotti, Y. Zhang, L. M. Hendershot, H. P. Harding, and D. Ron, "Dynamic interaction of $\mathrm{BiP}$ and ER stress transducers in the unfolded-protein response," Nature Cell Biology, vol. 2, pp. 326-332, 2000.

[26] J. Hassler, S. S. Cao, and R. J. Kaufman, "IRE1, a double-edged sword in pre-miRNA slicing and cell death," Developmental Cell, vol. 23, no. 5, pp. 921-923, 2012.

[27] X. Chen, J. Shen, and R. Prywes, "The luminal domain of ATF6 senses endoplasmic reticulum (ER) stress and causes translocation of ATF6 from the er to the Golgi," Journal of Biological Chemistry, vol. 277, no. 15, pp. 13045-13052, 2002.

[28] J. Wu, D. T. Rutkowski, and M. Dubois, "ATF6alpha optimizes long-term endoplasmic reticulum function to protect cells from chronic stress," Dev Cell, vol. 13, no. 3, pp. 351-364, 2007.

[29] K. Yamamoto, T. Sato, T. Matsui et al., "Transcriptional induction of mammalian ER quality control proteins is mediated by single or combined action of ATF $6 \alpha$ and XBP1," Developmental Cell, vol. 13, no. 3, pp. 365-376, 2007.

[30] T. Minamino, I. Komuro, and M. Kitakaze, "Endoplasmic reticulum stress as a therapeutic target in cardiovascular disease," Circulation Research, vol. 107, no. 9, pp. 1071-1082, 2010.

[31] M.-Q. Liu, Z. Chen, and L.-X. Chen, "Endoplasmic reticulum stress: a novel mechanism and therapeutic target for cardiovascular diseases," Acta Pharmacologica Sinica, vol. 37, no. 4, pp. 425-443, 2016.

[32] T. Minamino and M. Kitakaze, "ER stress in cardiovascular disease," Journal of Molecular and Cellular Cardiology, vol. 48, no. 6, pp. 1105-1110, 2010.

[33] S. Di Francescomarino, A. Sciartilli, V. Di Valerio, A. Di Baldassarre, and S. Gallina, "The effect of physical exercise on endothelial function," Sports Medicine, vol. 39, no. 10, pp. 797$812,2009$.

[34] J. Zhou, Š. Lhoták, B. A. Hilditch, and R. C. Austin, "Activation of the unfolded protein response occurs at all stages of atherosclerotic lesion development in apolipoprotein Edeficient mice," Circulation, vol. 111, no. 14, pp. 1814-1821, 2005.

[35] F. Urano, X. Wang, A. Bertolotti et al., "Coupling of stress in the ER to activation of JNK protein kinases by transmembrane protein kinase IRE1," Science, vol. 287, no. 5453, pp. 664-666, 2000.

[36] H. Nishitoh, A. Matsuzawa, K. Tobiume et al., "ASK1 is essential for endoplasmic reticulum stress-induced neuronal cell death triggered by expanded polyglutamine repeats," Genes and Development, vol. 16, no. 11, pp. 1345-1355, 2002.

[37] S. A. Oakes, S. S. Lin, and M. C. Bassik, "The control of endoplasmic reticulum-initiated apoptosis by the BCL-2 family of proteins," Current Molecular Medicine, vol. 6, no. 1, pp. 99109, 2006.
[38] M. C. Wei, W. X. Zong, E. H. Y. Cheng et al., "Proapoptotic BAX and BAK: a requisite gateway to mitochondrial dysfunction and death," Science, vol. 292, no. 5517, pp. 727-730, 2001.

[39] E. Szegezdi, D. C. MacDonald, T. N. Chonghaile, S. Gupta, and A. Samali, "Bcl-2 family on guard at the ER," American Journal of Physiology-Cell Physiology, vol. 296, no. 5, pp. C941-C953, 2009.

[40] J. M. Timmins, L. Ozcan, T. A. Seimon et al., "Calcium/ calmodulin-dependent protein kinase II links ER stress with Fas and mitochondrial apoptosis pathways," Journal of Clinical Investigation, vol. 119, no. 10, pp. 2925-2941, 2009.

[41] Y. Zhang and J. Ren, "Thapsigargin triggers cardiac contractile dysfunction via NADPH oxidase-mediated mitochondrial dysfunction: role of Akt dephosphorylation," Free Radical Biology and Medicine, vol. 51, no. 12, pp. 2172-2184, 2011.

[42] G. Li, C. Scull, L. Ozcan, and I. Tabas, "NADPH oxidase links endoplasmic reticulum stress, oxidative stress, and PKR activation to induce apoptosis," Journal of Cell Biology, vol. 191, no. 6, pp. 1113-1125, 2010.

[43] H. Li and U. Forstermann, "Uncoupling of endothelial NO synthase in atherosclerosis and vascular disease," Current Opinion in Pharmacology, vol. 13, no. 2, pp. 161-167, 2013.

[44] W. Zeng, Y.-H. Guo, W. Qi et al., "4-Phenylbutyric acid suppresses inflammation through regulation of endoplasmic reticulum stress of endothelial cells stimulated by uremic serum," Life Sciences, vol. 103, no. 1, pp. 15-24, 2014.

[45] M. Galán, M. Kassan, P. J. Kadowitz, M. Trebak, S. Belmadani, and K. Matrougui, "Mechanism of endoplasmic reticulum stress-induced vascular endothelial dysfunction," Biochimica et Biophysica Acta: Molecular Cell Research, vol. 1843, no. 6, pp. 1063-1075, 2014.

[46] J. Tao, W. Zhu, Y. Li et al., "Apelin-13 protects the heart against ischemia-reperfusion injury through inhibition of ERdependent apoptotic pathways in a time-dependent fashion," American Journal of Physiology - Heart and Circulatory Physiology, vol. 301, no. 4, pp. H1471-H1486, 2011.

[47] M. Shen, L. Wang, G. Yang et al., "Baicalin protects the cardiomyocytes from ER stress-induced apoptosis: inhibition of CHOP through induction of endothelial nitric oxide synthase," PLoS ONE, vol. 9, no. 2, p. e88389, 2014.

[48] H. Ichijo, E. Nishida, K. Irie et al., "Induction of apoptosis by ASK1, a mammalian MAPKKK that activates SAPK/JNK and p38 signaling pathways," Science, vol. 275, no. 5296, pp. 90-94, 1997.

[49] Q.-Y. Zheng, P.-P. Li, F.-S. Jin et al., "Ursolic acid induces ER stress response to activate ASK1-JNK signaling and induce apoptosis in human bladder cancer T24 cells," Cellular Signalling, vol. 25, no. 1, pp. 206-213, 2013.

[50] D. L. Eizirik, A. K. Cardozo, and M. Cnop, "The role for endoplasmic reticulum stress in diabetes mellitus," Endocrine Reviews, vol. 29, no. 1, pp. 42-61, 2008.

[51] J. Guo, Y. Bian, R. Bai, H. Li, M. Fu, and C. Xiao, "Globular adiponectin attenuates myocardial ischemia/reperfusion injury by upregulating endoplasmic reticulum $\mathrm{Ca}^{2+}$-Atpase activity and inhibiting endoplasmic reticulum stress," Journal of Cardiovascular Pharmacology, vol. 62, no. 2, pp. 143-153, 2013.

[52] X.-H. Liu, Z.-Y. Zhang, S. Sun, and X.-D. Wu, "Ischemic postconditioning protects myocardium from ischemia/reperfusion injury through attenuating endoplasmic reticulum stress," Shock, vol. 30, no. 4, pp. 422-427, 2008.

[53] B. Li, J. Tian, Y. Sun et al., "Activation of NADPH oxidase mediates increased endoplasmic reticulum stress and left ventricular 
remodeling after myocardial infarction in rabbits," Biochimica et Biophysica Acta - Molecular Basis of Disease, vol. 1852, no. 5, pp. 805-815, 2015.

[54] H.-S. Guan, H.-J. Shangguan, Z. Shang, L. Yang, X.-M. Meng, and S.-B. Qiao, "Endoplasmic reticulum stress caused by left ventricular hypertrophy in rats: effects of telmisartan," American Journal of the Medical Sciences, vol. 342, no. 4, pp. 318-323, 2011.

[55] C. S. Park, H. Cha, E. J. Kwon, P. K. Sreenivasaiah, and D. H. Kim, "The chemical chaperone 4-phenylbutyric acid attenuates pressure-overload cardiac hypertrophy by alleviating endoplasmic reticulum stress," Biochemical and Biophysical Research Communications, vol. 421, no. 3, pp. 578-584, 2012.

[56] S. Lee, Y. Park, M. Y. Zuidema, M. Hannink, and C. Zhang, "Effects of interventions on oxidative stress and inflammation of cardiovascular diseases," World Journal of Cardiology, vol. 3, pp. 18-24, 2011.

[57] T. Gotoh, M. Endo, and Y. Oike, "Endoplasmic reticulum stress-related inflammation and cardiovascular diseases," International Journal of Inflammation, vol. 2011, Article ID 259462, 8 pages, 2011.

[58] A.X. Zhou and I. Tabas, "The UPR in atherosclerosis," Seminars in Immunopathology, vol. 35, no. 3, pp. 321-332, 2013.

[59] P. Hu, Z. Han, A. D. Couvillon, R. J. Kaufman, and J. H. Exton, "Autocrine tumor necrosis factor alpha links endoplasmic reticulum stress to the membrane death receptor pathway through IRE1 $\alpha$-mediated NF- $\kappa \mathrm{B}$ activation and down-regulation of TRAF2 expression," Molecular \& Cellular Biology, vol. 26, no. 8, pp. 3071-3084, 2006.

[60] J. Deng, P. D. Lu, Y. Zhang et al., "Translational repression mediates activation of nuclear factor kappa B by phosphorylated translation initiation factor 2," Molecular and Cellular Biology, vol. 24, no. 23, pp. 10161-10168, 2004.

[61] H. Yamazaki, N. Hiramatsu, K. Hayakawa et al., "Activation of the Akt-NF- $\kappa$ B pathway by subtilase cytotoxin through the ATF6 branch of the unfolded protein response," Journal of Immunology, vol. 183, no. 2, pp. 1480-1487, 2009.

[62] Y. Li, R. F. Schwabe, T. DeVries-Seimon et al., "Free cholesterolloaded macrophages are an abundant source of tumor necrosis factor- $\alpha$ and interleukin-6: model of NF- $\kappa$ B- and map kinasedependent inflammation in advanced atherosclerosis," Journal of Biological Chemistry, vol. 280, no. 23, pp. 21763-21772, 2005.

[63] J. Yang, Y. Park, H. Zhang et al., "Feed-forward signaling of TNF-alpha and NF-kappaB via IKK-beta pathway contributes to insulin resistance and coronary arteriolar dysfunction in type 2 diabetic mice," The American Journal of Physiology-Heart and Circulatory Physiology, vol. 296, no. 6, pp. H1850-H1858, 2009.

[64] Y. Park, J. Yang, H. Zhang, X. Chen, and C. Zhang, "Effect of PAR2 in regulating TNF- $\alpha$ and $\mathrm{NAD}(\mathrm{P}) \mathrm{H}$ oxidase in coronary arterioles in type 2 diabetic mice," Basic Research in Cardiology, vol. 106, no. 1, pp. 111-123, 2011.

[65] Y. Park, R. D. Prisby, B. J. Behnke et al., "Effects of aging, TNF$\alpha$, and exercise training on angiotensin II-induced vasoconstriction of rat skeletal muscle arterioles," Journal of Applied Physiology, vol. 113, no. 7, pp. 1091-1100, 2012.

[66] J. Yang, Y. Park, H. Zhang et al., "Role of MCP-1 in tumor necrosis factor- $\alpha$-induced endothelial dysfunction in type 2 diabetic mice," American Journal of Physiology - Heart and Circulatory Physiology, vol. 297, no. 4, pp. H1208-H1216, 2009.

[67] M. Karaduman, A. Sengul, C. Oktenli et al., "Tissue levels of adiponectin, tumour necrosis factor-alpha, soluble intercellular adhesion molecule-1 and heart-type fatty acid-binding protein in human coronary atherosclerotic plaques," Clinical Endocrinology, vol. 64, no. 2, pp. 196-202, 2006.

[68] I. Petrache, K. Birukov, A. L. Zaiman et al., "Caspase-dependent cleavage of myosin light chain kinase (MLCK) is involved in TNF- $\alpha$-mediated bovine pulmonary endothelial cell apoptosis," FASEB Journal, vol. 17, no. 3, pp. 407-416, 2003.

[69] S. Arai, N. Harada, N. Kubo et al., "Induction of inducible nitric oxide synthase and apoptosis by LPS and TNF- $\alpha$ in nasal microvascular endothelial cells," Acta Oto-Laryngologica, vol. 128, no. 1, pp. 78-85, 2008.

[70] Z. Zhou, P. Gengaro, W. Wang et al., "Role of NF- $\kappa$ B and PI 3-kinase/Akt in TNF- $\alpha$-induced cytotoxicity in microvascular endothelial cells," American Journal of Physiology - Renal Physiology, vol. 295, no. 4, pp. F932-F941, 2008.

[71] M. Galán, M. Kassan, S.-K. Choi et al., "A novel role for epidermal growth factor receptor tyrosine kinase and its downstream endoplasmic reticulum stress in cardiac damage and microvascular dysfunction in type 1 diabetes mellitus," Hypertension, vol. 60, no. 1, pp. 71-80, 2012.

[72] H. Wen, E. A. Miao, and J. P.-Y. Ting, "Mechanisms of NODlike receptor-associated inflammasome activation," Immunity, vol. 39, no. 3, pp. 432-441, 2013.

[73] S. Kim, Y. Joe, S. O. Jeong et al., "Endoplasmic reticulum stress is sufficient for the induction of IL-1beta production via activation of the NF-kappaB and inflammasome pathways," Innate Immunity, vol. 20, no. 8, pp. 799-815, 2014.

[74] A. G. Lerner, J. P. Upton, P. V. K. Praveen et al., "IRE1 $\alpha$ induces thioredoxin-interacting protein to activate the NLRP3 inflammasome and promote programmed cell death under irremediable ER stress," Cell Metabolism, vol. 16, no. 2, pp. 250264, 2012.

[75] I. N. Mohamed, S. S. Hafez, A. Fairaq, A. Ergul, J. D. Imig, and A. B. El-Remessy, "Thioredoxin-interacting protein is required for endothelial NLRP3 inflammasome activation and cell death in a rat model of high-fat diet," Diabetologia, vol. 57, no. 2, pp. 413-423, 2014.

[76] S. Blankenberg, T. Godefroy, O. Poirier et al., "Haplotypes of the caspase-1 gene, plasma caspase-1 levels, and cardiovascular risk," Circulation Research, vol. 99, no. 1, pp. 102-108, 2006.

[77] P. Kleinbongard, G. Heusch, and R. Schulz, "TNF $\alpha$ in atherosclerosis, myocardial ischemia/reperfusion and heart failure," Pharmacology and Therapeutics, vol. 127, no. 3, pp. 295314, 2010.

[78] Y. Li, J. Yang, M.-H. Chen et al., "Ilexgenin A inhibits endoplasmic reticulum stress and ameliorates endothelial dysfunction via suppression of TXNIP/NLRP3 inflammasome activation in an AMPK dependent manner," Pharmacological Research, vol. 99, pp. 101-115, 2015.

[79] J. Li, Y. Wang, Y. Wang et al., "Pharmacological activation of AMPK prevents Drpl-mediated mitochondrial fission and alleviates endoplasmic reticulum stress-associated endothelial dysfunction," Journal of Molecular and Cellular Cardiology, vol. 86, pp. 62-74, 2015.

[80] S. B. Cullinan and J. A. Diehl, "Coordination of ER and oxidative stress signaling: the PERK/Nrf2 signaling pathway," International Journal of Biochemistry and Cell Biology, vol. 38, no. 3, pp. 317-332, 2006.

[81] H. Y. Jiang, S. A. Wek, B. C. McGrath et al., "Phosphorylation of the $\alpha$ subunit of eukaryotic initiation factor 2 is required for activation of NF- $\kappa \mathrm{B}$ in response to diverse cellular stresses," Molecular and Cellular Biology, vol. 23, no. 16, pp. 5651-5663, 2003. 
[82] S. Lee, Y. Park, K. C. Dellsperger, and C. Zhang, "Exercise training improves endothelial function via adiponectindependent and independent pathways in type 2 diabetic mice," The American Journal of Physiology-Heart and Circulatory Physiology, vol. 301, no. 2, pp. H306-H314, 2011.

[83] S.-K. Choi, M. Lim, S.-I. Yeon, and Y.-H. Lee, "Inhibition of endoplasmic reticulum stress improves coronary artery function in type 2 diabetic mice," Experimental Physiology, vol. 101, no. 6, pp. 768-777, 2016.

[84] S.-K. Choi, M. Lim, S.-H. Byeon, and Y.-H. Lee, "Inhibition of endoplasmic reticulum stress improves coronary artery function in the spontaneously hypertensive rats," Scientific Reports, vol. 6, Article ID 31925, p. 31925, 2016.

[85] Y. Park, S. Capobianco, X. Gao, J. R. Falck, K. C. Dellsperger, and C. Zhang, "Role of EDHF in type 2 diabetes-induced endothelial dysfunction," American Journal of Physiology Heart and Circulatory Physiology, vol. 295, no. 5, pp. H1982H1988, 2008.

[86] Y. Park, J. Wu, H. Zhang, Y. Wang, and C. Zhang, "Vascular dysfunction in type 2 diabetes: emerging targets for therapy," Expert Review of Cardiovascular Therapy, vol. 7, no. 3, pp. 209213, 2009.

[87] L. H. M. Bozi, P. R. Jannig, N. Rolim et al., "Aerobic exercise training rescues cardiac protein quality control and blunts endoplasmic reticulum stress in heart failure rats," Journal of Cellular and Molecular Medicine, vol. 20, no. 11, pp. 2208-2212, 2016.

[88] W. S. Cheang, W. T. Wong, L. Zhao et al., "PPARdelta Is required for exercise to attenuate endoplasmic reticulum stress and endothelial dysfunction in diabetic mice," Diabetes, vol. 66, no. 2, pp. 519-528, 2017.

[89] J. Kang, "Exercise copes with prolonged stress-induced impairment of spatial memory performance by endoplasmic reticulum stress," The Journal of Exercise Nutrition and Biochemistry, vol. 19, no. 3, pp. 191-197, 2015.

[90] L. Deldicque, P. D. Cani, N. M. Delzenne, K. Baar, and M. Francaux, "Endurance training in mice increases the unfolded protein response induced by a high-fat diet," Journal of Physiology and Biochemistry, vol. 69, no. 2, pp. 215-225, 2013.

[91] B. C. Pereira, A. L. Da Rocha, A. P. Pinto et al., "Excessive eccentric exercise-induced overtraining model leads to endoplasmic reticulum stress in mice skeletal muscles," Life Sciences, vol. 145, pp. 144-151, 2016.

[92] K. Kim, Y.-H. Kim, S.-H. Lee, M.-J. Jeon, S.-Y. Park, and K.-O. Doh, "Effect of exercise intensity on unfolded protein response in skeletal muscle of rat," Korean Journal of Physiology and Pharmacology, vol. 18, no. 3, pp. 211-216, 2014.

[93] S. S. Lee, J. H. Yoo, and Y. S. So, "Effect of the low-versus highintensity exercise training on endoplasmic reticulum stress and GLP-1 in adolescents with type 2 diabetes mellitus," Journal of Physical Therapy Science, vol. 27, no. 10, pp. 3063-3068, 2015.

[94] Y. Kim, M. Park, S. Boghossian, and D. A. York, “Three weeks voluntary running wheel exercise increases endoplasmic reticulum stress in the brain of mice," Brain Research, vol. 1317, pp. 13-23, 2010.

[95] Z. Murlasits, Y. Lee, and S. K. Powers, "Short-term exercise does not increase ER stress protein expression in cardiac muscle," Medicine and Science in Sports and Exercise, vol. 39, no. 9, pp. 1522-1528, 2007.

[96] E. R. Ropelle, M. B. Flores, D. E. Cintra et al., "IL-6 and IL10 anti-inflammatory activity links exercise to hypothalamic insulin and leptin sensitivity through IKKbeta and ER stress inhibition," PLoS Biology, vol. 8, 2010.

[97] D. I. Ogborn, B. R. McKay, J. D. Crane, G. Parise, and M. A. Tarnopolsky, "The unfolded protein response is triggered following a single, unaccustomed resistance-exercise bout," American Journal of Physiology - Regulatory Integrative and Comparative Physiology, vol. 307, no. 6, pp. R664-R669, 2014.

[98] J. Wu, J. L. Ruas, J. L. Estall et al., “The unfolded protein response mediates adaptation to exercise in skeletal muscle through a PGC-1alpha/ATF6alpha complex," Cell Metab, vol. 13, no. 2, pp. 160-169, 2011.

[99] H. J. Kim, C. Jamart, L. Deldicque et al., "Endoplasmic reticulum stress markers and ubiquitin-proteasome pathway activity in response to a $200 \mathrm{~km}$ run," Medicine and Science in Sports and Exercise, vol. 43, no. 1, pp. 18-25, 2011.

[100] J. M. Memme, A. N. Oliveira, and D. A. Hood, "Chronology of UPR activation in skeletal muscle adaptations to chronic contractile activity," American Journal of Physiology - Cell Physiology, vol. 310, no. 11, pp. C1024-C1036, 2016. 


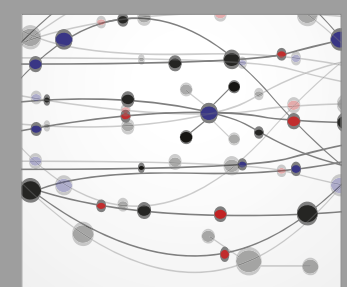

The Scientific World Journal
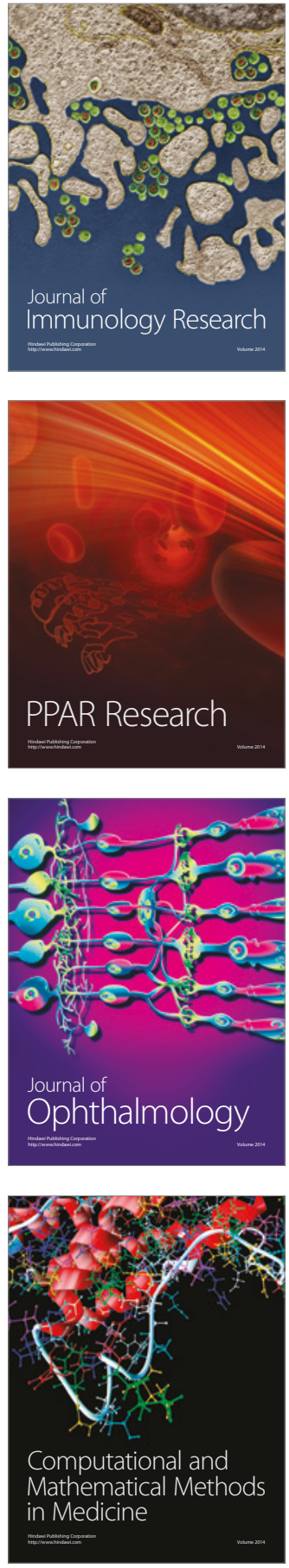

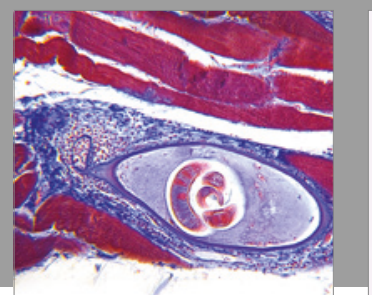

Gastroenterology Research and Practice
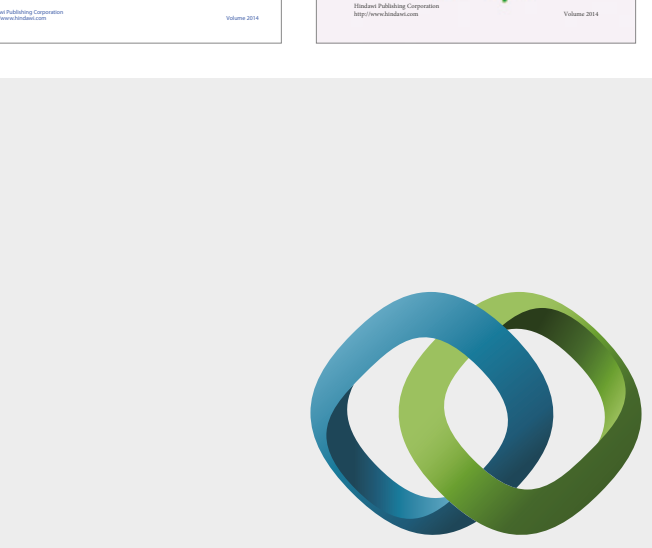

\section{Hindawi}

Submit your manuscripts at

https://www.hindawi.com
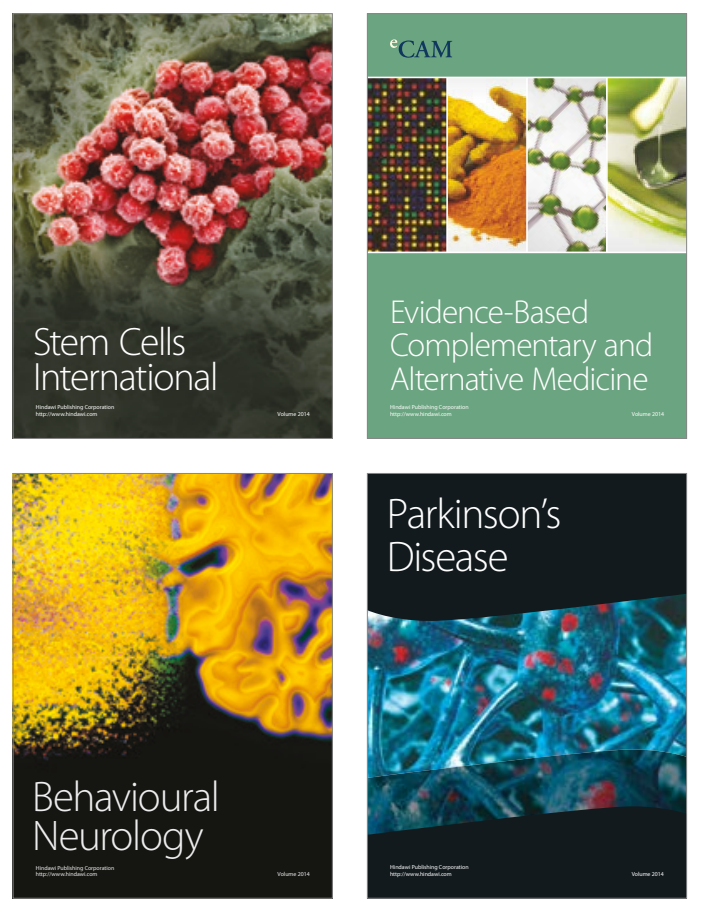
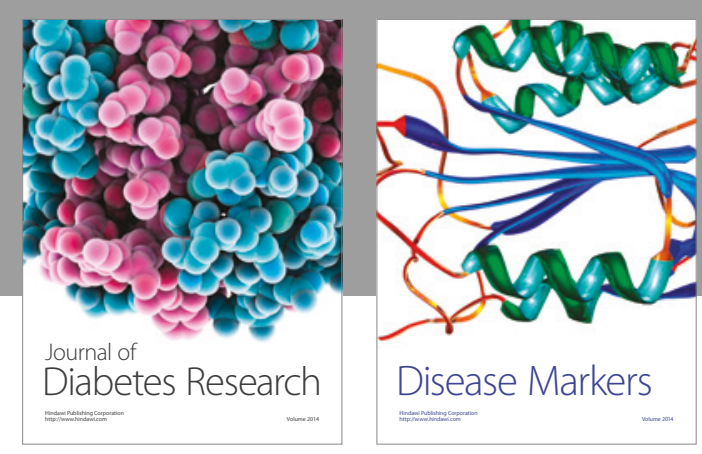

Disease Markers
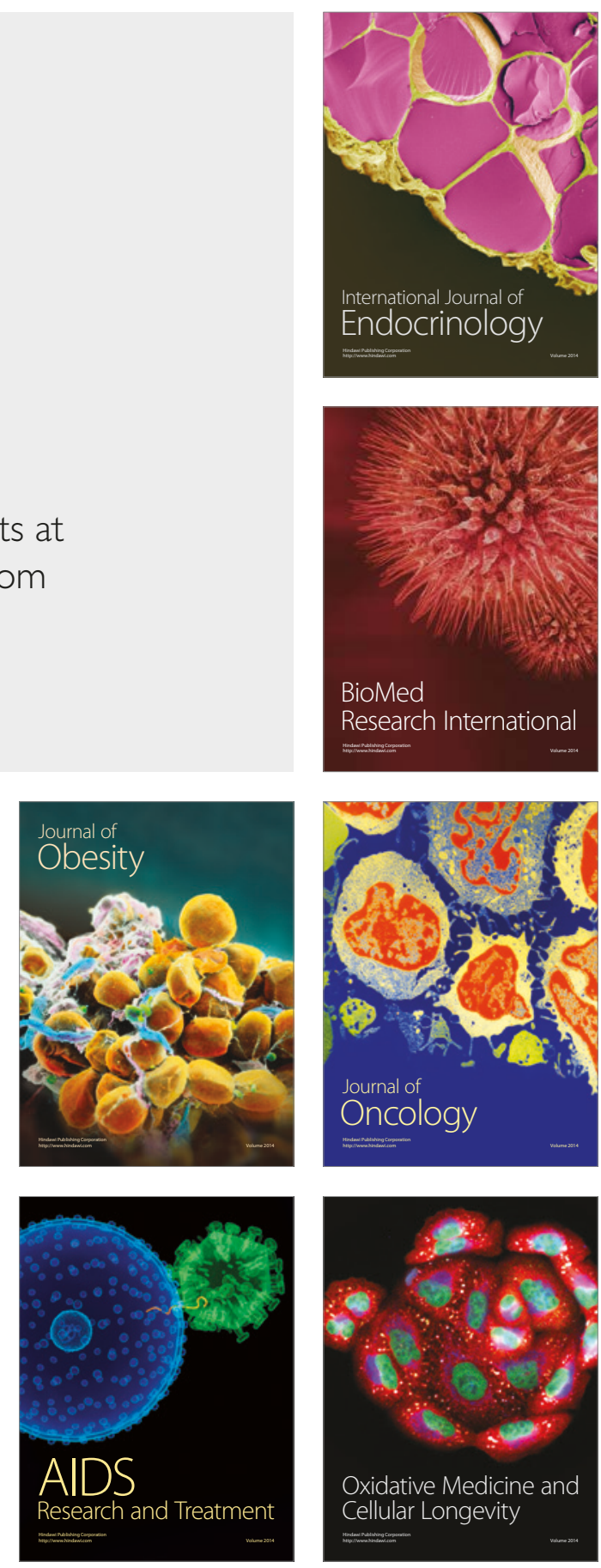